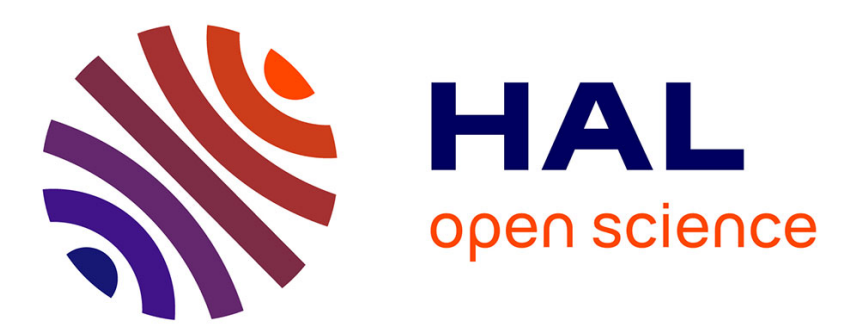

\title{
A time-delay estimation approach for coherent GPR signals by taking into account the noise pattern and radar pulse
}

Jingjing Pan, Meng Sun, Yide Wang, Cédric Le Bastard, Vincent Baltazart

\section{- To cite this version:}

Jingjing Pan, Meng Sun, Yide Wang, Cédric Le Bastard, Vincent Baltazart. A time-delay estimation approach for coherent GPR signals by taking into account the noise pattern and radar pulse. Signal Processing, 2020, 176, pp.107654. 10.1016/j.sigpro.2020.107654 . hal-02569685

\section{HAL Id: hal-02569685 \\ https://hal.science/hal-02569685}

Submitted on 29 Jun 2020

HAL is a multi-disciplinary open access archive for the deposit and dissemination of scientific research documents, whether they are published or not. The documents may come from teaching and research institutions in France or abroad, or from public or private research centers.
L'archive ouverte pluridisciplinaire HAL, est destinée au dépôt et à la diffusion de documents scientifiques de niveau recherche, publiés ou non, émanant des établissements d'enseignement et de recherche français ou étrangers, des laboratoires publics ou privés. 


\title{
A time-delay estimation approach for coherent GPR signals by taking into account the noise pattern and radar pulse
}

\author{
Jingjing Pan ${ }^{\mathrm{a}}$, Meng Sun ${ }^{\mathrm{a}}$, Yide Wang ${ }^{\mathrm{b}}$, Cédric Le Bastard, Vincent Baltazart $^{\mathrm{c}}$ \\ ${ }^{a}$ Department of Electronic Engineering, Nanjing University of Aeronautics and Astronautics, Nanjing, People's Republic of \\ China, 211106 \\ Email:(jingjingpan@nuaa.edu.cn) \\ ${ }^{b}$ Institut d'Electronique et Télécommunications de Rennes (IETR), Université de Nantes, UMR CNRS 6164, Rue Christian \\ Pauc BP 50609, Nantes 44306, France \\ ${ }^{c}$ The French Institute of Science and Technology for Transport, Development and Networks (IFSTTAR), Route de la \\ Bouaye, 44340, Bouguenais, France
}

\begin{abstract}
In this paper, we propose to perform time-delay estimation (TDE) of coherent GPR signals by taking into accout the radar pulse and noise pattern. The proposed method can process raw GPR data without whitening procedure and decorrelate coherent signals properly with a Hankel matrix which contains the signal eigenvector, the radar pulse and the noise characteristics. Simulations and a field experiment show the effectiveness of the proposed method.
\end{abstract}

Keywords: Time-delay estimation (TDE), ground penetrating radar (GPR), coherent signals

\section{Introduction}

In civil engineering, ground penetrating radar (GPR) is a widely used non-destructive device in subsurface sensing in many aspects, such as building structure and quality assessment, buried targets localization and so on $[1,2]$. In the survey of horizontally stratified media, for example, pavement, time-delay estimation 5 (TDE) is important that provides structural information by using GPR [3, 4]. High resolution methods, including multiple signal classification (MUSIC) [5] and estimation of signal parameters via rational invariance technique (ESPRIT) [6], are popular in parameter estimation, especially for thin layer pavements.

Nevertheless, most of the TDE algorithms assume ideal Dirac radar pulse, making the temporal whitening of the GPR data indispensable. The whitening procedure, dividing the raw data by the radar pulse, modifies implicitly the properities of the noise and might therefore affect the backscattered echoes through the signal to noise ratio (SNR) [7].

The authors in $[7,8]$ propose to take the radar pulse into account in the estimation. The work in [8] supposes to deal with Gaussian white noise. While [7] considers any noise pattern but assumes null correlation among the backscattered echoes, which is not valid in practical GPR applications. There are 
also a family of sparse deconvolution methods in TDE, for example, orthogonal matching pursuit (OMP) method [9], $l_{1}$-norm deconvolution method [10,11], without restrictions on the radar pulse or the noise. However, OMP shows poor performance in terms of resolution power while $l_{1}$-norm deconvolution method has high computational burden and suffers performance degradation in low SNR scenarios, especially with weak signals [11].

The radar pulse leads to a nonlinear frequency behavior in the exponent of the measured GPR echoes. Therefore, the conventional spatial smoothing preprocessing (SSP) and its variants $[12,13]$ can't be applied directly for the decorrelation, since they are based on the linear frequency behavior in the exponent of the received signals. Besides, there are several methods constructing new matrices to decorrelate coherent signals. In [14], the authors build a Toeplitz matrix with the elements in the data covariance matrix, whose 25 rank is independent of the coherency among signals. The structure of Hankel matrix is also exploited to decorrelate signals with enhanced performance $[15,16]$. The construction of the Hankel matrix in [15] is based on the received data while that in $[16]$ is based on the data covariance matrix. In this correspondence, we would like to modify [7] for coherent scenarios by using the matrix reconstruction approach and considering both the noise pattern and radar pulse. The proposed method has the following merits: 1) it can directly deal with raw GPR data without whitening procedure; 2) the decorrelation is achieved through a constructed Hankel matrix which contains the signal eigenvector, the radar pulse and the noise characteristics; 3) it has enhanced performance especially for weak signals.

The rest of this paper is organized as follows. Section 2 gives the signal model. Section 3 describes the proposed method. Section 4 presents the simulation results along with a field experiment. Conclusions are 35 drawn in Section 5.

\section{Signal Model}

The stratified non-dispersive media model in [7] is adopted in this paper. Assume the media is composed of $K$ interfaces and the GPR observations are conducted with $M$ equispaced frequencies. Therefore, the backscattered echoes are time-shifted and attenuated replicas of the transmitted signal. Within frequency bandwidth $B$, the backscattered echoes in vector form can be written as

$$
\mathbf{r}=\mathbf{\Lambda A s}+\mathbf{n}
$$

with the following notations: 
- $\mathbf{r}=\left[r\left(f_{1}\right), \ldots, r\left(f_{M}\right)\right]^{T}$ is the $M \times 1$ received GPR signal vector in frequency domain; $f_{m}=f_{1}+(m-$ 1) $\Delta f$, with $f_{1}$ the beginning of bandwidth and $\Delta f$ the frequency shift, $m=1, \ldots, M$; superscript $T$ denotes transpose operation;

- $\mathbf{s}=\left[s_{1}, \ldots, s_{K}\right]^{T}$ is the $K \times 1$ source vector composed of echoes' amplitudes;

- $\mathbf{n}=\left[n_{1}, \ldots, n_{M}\right]^{T}$ is the $M \times 1$ noise vector with zero mean;

- $\boldsymbol{\Lambda}=\operatorname{diag}\left\{\tilde{e}_{1}, \ldots, \tilde{e}_{M}\right\}$ is a $M \times M$ diagonal matrix with diagonal elements corresponding to the Fourier transform of the radar pulse;

- $\mathbf{A}=\left[\mathbf{a}\left(\tau_{1}\right), \ldots, \mathbf{a}\left(\tau_{K}\right)\right]$ is the mode matrix whose columns are defined as $\mathbf{a}\left(\tau_{k}\right)=\left[e^{-j 2 \pi f_{1} \tau_{k}}, \ldots, e^{-j 2 \pi f_{M} \tau_{k}}\right]^{T}$, $k=1, \ldots, K ; \tau_{k}$ is the time of arrival of the $k$ th echo.

Assuming the noise to be independent of the backscattered echoes, the data covariance matrix of $\mathbf{r}$ can be expressed as

$$
\mathbf{R}=E\left(\mathbf{r r}^{H}\right)=\mathbf{\Lambda} \mathbf{A} \mathbf{R}_{s} \mathbf{A}^{H} \boldsymbol{\Lambda}^{H}+\sigma^{2} \mathbf{\Sigma}
$$

where $E($.$) denotes the ensemble average; \mathbf{R}_{s}=E\left(\mathbf{s s}^{H}\right)$ and $\sigma^{2} \boldsymbol{\Sigma}$ are the signal sources and noise covariance matrix, respectively; superscript $H$ denotes transpose conjugate operation. In this paper, the signal model 55 is under the following assumptions:

1. The backscattered echoes are coherent; the signal covariance matrix $\mathbf{R}_{s}$ is rank one;

2. The noise is statistically independent, zero mean complex Gaussian random process, and can be spatially white or colored;

3. The radar pulse $\boldsymbol{\Lambda}$ and noise covariance matrix $\boldsymbol{\Sigma}$ are known beforehand;

4. The noise is independent of all the backscattered echoes.

\section{Methodology}

\subsection{Generalized eigenvalue decomposition}

The generalized eigenvalue decomposition $(\operatorname{GEVD})$ of the matrix pair $(\mathbf{R}, \boldsymbol{\Sigma})$ is defined as in $[17,18]$

$$
\mathbf{R e}_{m}=\lambda_{m} \boldsymbol{\Sigma} \mathbf{e}_{m}, m=1, \ldots, M
$$


with $\lambda_{m}$ the $m$ th eigenvalue in descending order and $\mathbf{e}_{m}$ the corresponding eigenvector with dimension ${ }_{65} M \times 1$.

In contrast to [7], the backscattered GPR echoes in this paper are coherent. In this case, the eigenvalues after the generalized eigenanalysis are distributed as $\lambda_{1}>\lambda_{2}=\cdots=\lambda_{M}=\sigma^{2}$.

Referring to the largest eigenvalue $\lambda_{1}$, (3) can be expressed as

$$
\mathbf{R e}_{1}=\lambda_{1} \boldsymbol{\Sigma} \mathbf{e}_{1}
$$

Substituting (2) into (4) and after some mathematical manipulations, we deduce

$$
\mathbf{A R}_{s} \mathbf{A}^{H} \boldsymbol{\Lambda}^{H} \mathbf{e}_{1}=\left(\lambda_{1}-\sigma^{2}\right) \boldsymbol{\Lambda}^{-1} \mathbf{\Sigma} \mathbf{e}_{1}
$$

Let $\mathbf{t}=\mathbf{R}_{s} \mathbf{A}^{H} \boldsymbol{\Lambda}^{H} \mathbf{e}_{1}$, (5) can be reformulated as follows:

$$
\mathbf{g}=\mathbf{A t}=\left(\lambda_{1}-\sigma^{2}\right) \mathbf{\Lambda}^{-1} \mathbf{\Sigma} \mathbf{e}_{1}
$$

where $\mathbf{t}$ is a $K \times 1$ vector whose elements are non-zero; $\mathbf{g}$ is a $M \times 1$ vector.

\subsection{Proposed method for TDE}

In $[19,20]$, the authors only make use of the signal eigenvector $\mathbf{e}_{1}$ in the decorrelation with enhanced performance. It implies that in coherent scenarios, the signal eigenvector corresponding to the largest eigenvalue contains all the information of signals and can be used for decorrelation. Unlike [19, 20], we propose to exploit not only the information of $\mathbf{e}_{1}$ but also the radar pulse and noise characteristics to decorrelate the coherent backscattered echoes, which is achieved by transforming $\mathbf{g}$ into a Hankel matrix $\mathbf{X}$ as in $[15,16],[21]$ :

$$
\mathbf{X}=\left[\begin{array}{cccc}
g_{1} & g_{2} & \ldots & g_{L} \\
g_{2} & g_{3} & \ldots & g_{L+1} \\
\vdots & \vdots & & \vdots \\
g_{M-L+1} & g_{M-L+2} & \ldots & g_{M}
\end{array}\right]_{(M-L+1) \times L}
$$

where $g_{m}$ is the $m$ th element of vector $\mathbf{g}, m=1, \ldots, M$ and $K \leq L \leq M-K+1$.

Matrix $\mathbf{X}$ in (7) can be reformulated as

$$
\mathbf{X}=\mathbf{A}_{1} \mathbf{T} \mathbf{A}_{2}^{T}
$$


where

$$
\begin{gathered}
\mathbf{A}_{1}=\left[\begin{array}{cccc}
1 & 1 & \ldots & 1 \\
z_{1} & z_{2} & \ldots & z_{K} \\
\vdots & \vdots & & \vdots \\
z_{1}^{M-L} & z_{2}^{M-L} & \ldots & z_{K}^{M-L}
\end{array}\right]_{(M-L+1) \times K} \\
\mathbf{A}_{2}=\left[\begin{array}{cccc}
1 & 1 & \ldots & 1 \\
z_{1} & z_{2} & \ldots & z_{K} \\
\vdots & \vdots & & \vdots \\
z_{1}^{L-1} & z_{2}^{L-1} & \ldots & z_{K}^{L-1}
\end{array}\right]_{L \times K},
\end{gathered}
$$

and $\mathbf{T}=\operatorname{diag}\left\{e^{-j 2 \pi f_{1} \tau_{1}} t_{1}, e^{-j 2 \pi f_{1} \tau_{2}} t_{2}, \ldots, e^{-j 2 \pi f_{1} \tau_{K}} t_{K}\right\}$ with $z_{k}=e^{-j 2 \pi \Delta f \tau_{k}}$ and $t_{k}$ the $k$ th element of $\mathbf{t}$.

Matrices $\mathbf{A}_{1}$ and $\mathbf{A}_{2}$ have Vandermonde structure and they are therefore non-singular. We can also prove that $\mathbf{T}$ is of full rank, since $t_{k}$ and $e^{-j 2 \pi f_{1} \tau_{k}}$ are non-zero. The rank of matrix $\mathbf{X}$ is $K$, which is not 75 affected by the coherency. In this case, the truncated singular value decomposition (SVD) of matrix $\mathbf{X}$ is

$$
\mathbf{X}=\mathbf{U}_{s} \Phi_{s} \mathbf{V}_{s}^{H}
$$

where $\boldsymbol{\Phi}_{s}$ is a diagonal matrix whose elements are the $K$ largest non-zero singular values of $\mathbf{X} ; \mathbf{U}_{s}$ and $\mathbf{V}_{s}$ are respectively the left and right singular vector matrices of $\mathbf{X}$, corresponding to the $K$ dominant singular values. Define the singular vector matrices associated with the remaining smaller singular values as $\mathbf{U}_{n}$ and $\mathbf{V}_{n}$, respectively. We can multiply (11) by $\mathbf{V}_{n}$ under the concept of orthogonality:

$$
\mathbf{X} \mathbf{V}_{n}=\mathbf{U}_{s} \Phi_{s} \mathbf{V}_{s}^{H} \mathbf{V}_{n}=\mathbf{0}
$$

Substituting (8) into (12) yields:

$$
\mathbf{X} \mathbf{V}_{n}=\mathbf{A}_{1} \mathbf{T} \mathbf{A}_{2}^{T} \mathbf{V}_{n}=\mathbf{0}
$$

Since $\mathbf{A}_{1}$ is a Vandermonde matrix and $\mathbf{T}$ is non-singular, the above formula implies:

$$
\mathbf{A}_{2}^{H} \mathbf{V}_{n}^{*}=\mathbf{0}
$$

$80 \quad$ where $*$ denotes conjugate operation.

According to (14), the time-delays can be estimated by finding the zeros of $\overline{\mathbf{a}}(\tau) \mathbf{V}_{n}^{*}$, with $\overline{\mathbf{a}}(\tau)=$ 
$\left[1, e^{-j 2 \pi \Delta f \tau}, \ldots, e^{-j 2 \pi \Delta f(L-1) \tau}\right]^{H}$. In theory, it is identical to evaluate the polynomial $D(z)$ on the unit circle, like in Root-MUSIC [22]. The expression of $D(z)$ is

$$
D(z)=\sum_{l=-L+1}^{L-1} \alpha_{l} z^{-l}
$$

where $\alpha_{l}$ is the sum of entries of $\mathbf{P}$ along the $l$ th diagonal and $\mathbf{P}=\mathbf{V}_{n}^{*} \mathbf{V}_{n}^{T}$. The time-delays can be derived by the $K$ roots that are inside and closest to the unit circle.

Matrix $\mathbf{U}_{n}$ can also be applied in TDE but with similar performance, which is not included here. In addition, $\mathbf{X}$ can be extended into a forward-backward version as in [13]:

$$
\mathbf{Y}=\left[\begin{array}{c}
\mathbf{X} \\
\mathbf{J}_{1} \mathbf{X}^{*} \mathbf{J}_{2}
\end{array}\right]
$$

where $\mathbf{J}_{1}$ and $\mathbf{J}_{2}$ are anti-identity matrices with dimensions $(M-L+1) \times(M-L+1)$ and $L \times L$, respectively. The other estimation steps with matrix $\mathbf{Y}$ are the same as those with $\mathbf{X}$. In the following, the estimation ${ }_{85}$ with matrices $\mathbf{X}$ and $\mathbf{Y}$ are referred as the 'Proposed method-F' and 'Proposed method-FB', respectively.

To sum up, the proposed procedures are as follows:

1. Estimate the data covariance matrix R;

2. Perform GEVD on the matrix pair $(\mathbf{R}, \boldsymbol{\Sigma})$;

3. Calculate $\mathbf{g}$ according to $(6)$;

4. Construct matrices $\mathbf{X}$ and $\mathbf{Y}$ and apply SVD on them;

5. Build polynomial $D(z)$ and estimate the time-delays with the roots inside and closest to the unit circle.

\section{Numerical and experimental results}

In order to evaluate the performance of the proposed method, several simulations and an experiment are carried out in this section.

In the simulations, the stratified medium is assumed to have three layers, as shown in Figure 1. The dielectric constants of these layers are $\epsilon_{1}=3, \epsilon_{2}=8$ and $\epsilon_{3}=9$, respectively. The thickness of Layers 1 and 2 are $H_{1}=25 \mathrm{~mm}$ and $H_{2}=50 \mathrm{~mm}$, respectively. The frequency range of the step frequency GPR is $B=2 \mathrm{GHz}$, with $f \in[1,3] \mathrm{GHz}$ and $M=21$ frequency samples. The amplitudes of backscattered echoes are respectively $S_{1}=0.267, S_{2}=0.223$ and $S_{3}=0.026$, with the corresponding time of arrivals $\left[\tau_{1}, \tau_{2}, \tau_{3}\right]=$ 
For a given bandwidth $B$, the time resolution of a technique is defined as the minimal time interval $\Delta \tau$ between two distinguishable echoes [3]. Conventional FFT-based methods are restricted to distinguish non-overlapping echoes, and its time resolution is about $B \Delta \tau=1$. The time-delays within Layers 1 and 2 are $\Delta \tau_{1}=\tau_{2}-\tau_{1}=0.29 \mathrm{~ns}, \Delta \tau_{2}=\tau_{3}-\tau_{2}=0.94 \mathrm{~ns}$. In consequence, the corresponding products are $B \Delta \tau_{1}=0.58$ and $B \Delta \tau_{2}=1.88$, which means $S_{0}$ and $S_{1}$ are overlapped while $S_{1}$ and $S_{2}$ are not. $L$ is set as 11.

The relative-root-mean-square-error (RRMSE) is used to evaluate the statistical performance of the proposed method:

$$
\operatorname{RRMSE}=\frac{\sqrt{\frac{1}{Q} \sum_{q=1}^{Q}\left(\widehat{\Delta \tau}_{k q}-\Delta \tau_{k}\right)^{2}}}{\Delta \tau_{k}}
$$

where $\widehat{\Delta \tau}_{k q}$ denotes the estimate of the time-delay within the $k$ th layer for the $q$ th run; $\Delta \tau_{k}$ is the true value; $Q$ is the number of Monte Carlo trials.

The radar pulse is modeled as a Ricker pulse in this paper, which is a common pattern in GPR [7]. During the Monte Carlo process, the colored noise is generated as in [23]:

$$
\sigma^{2} \boldsymbol{\Sigma}=10^{-\frac{\mathrm{SNR}}{20}} \frac{\mathbf{G G}^{H}}{\left\|\mathbf{G G}^{H}\right\|}
$$

where the elements of matrix $\mathbf{G}$ with dimension $M \times M$ are generated with a sequence of independent and identically distributed zero mean gaussian random variables with unit standard deviation.

Figures 2 and 3 show the TDE results for $\Delta \tau_{1}$ and $\Delta \tau_{2}$ with respect to SNR through 200 Monte Carlo trials, respectively. The data covariance matrix is estimated with 500 snapshots. The estimates of RootMUSIC with MSSP and a second-order cone programming (SOCP) based $l_{1}$-norm deconvolution method are recorded for comparison, called Root-MUSIC-MSSP and $l_{1}$-norm, respectively. In order to apply MSSP, the echoes are whitened by the radar pulse. The size of elements in the sub-band of MSSP is 11, equal to L. In the comparison, Root-MUSIC-MSSP doesn't take into account the colored noise. Given the noise distribution and SNR, the optimal regularization parameter of the $l_{1}$-norm method is set according to [11].

As shown in Figures 2 and 3, the RRMSEs of all the methods are descreasing with the increasing of SNR. The statistical curves are closer to each other in the estimation of the first time-delay, than those of the second time-delay. It's specially true for the curves of $l_{1}$-norm. The reason may lie in the presence of weak signal, since the amplitude of the third echo is only 0.026 while the other two are 0.267 and 0.223 , respectively. The proposed method with forward only operation and Root-MUSIC-MSSP are less effective in TDE, compared with the proposed method with both forward and backward operation, no matter $B \Delta \tau$ 
is greater than 1 or not. The proposed method shows enhanced performance by considering the radar pulse 125 and the noise, especially for weak signals.

In the second simulation, we evaluate the accuracy of the proposed method in terms of the number of snapshots. SNR is $15 \mathrm{~dB}$. The other settings are the same with the first simulation. In Figures 4 and 5 , the RRMSEs are getting smaller when the number of snapshots gets larger. The proposed method with forward and backward operation outperforms the others for each number of snapshots. In Figure 4, the curves of the proposed method with forward operation, the $l_{1}$-norm method and Root-MUSIC-MSSP are similar when the number of snapshots is small; when the number of snapshots increases, larger than 600 in this case, the proposed method with forward operation achieves better result than Root-MUSIC-MSSP and $l_{1}$-norm. Similar to Figures 2 and $3, l_{1}$-norm has smaller RRMSEs in the estimation of $\Delta \tau_{1}$ than that of $\Delta \tau_{2}$, for each number of snapshots.

Apart from the simulations, the proposed method is tested with a field experiment on IFSTTAR fatigue carousel [24]. The IFSTTAR's pavement fatigue carrousel is a circular outdoor test track which allows various loading capabilities and speeds (refer to Fig. 1 in [24] for more details). There are three different types of artificial debondings: sand, geotextile and uncoated interface. In this experiment, only the measurements on the sand zone are used. The probed pavement is composed of two layers of asphalt, whose structure is shown in Figure 6. The observation radar system is composed of a vector network analyzer (VNA) and a quasi-monostatic step frequency radar with closely located transmitter $(\mathrm{Tx})$ and receiver $(\mathrm{Rx})$. The distance between $\mathrm{Tx}$ and $\mathrm{Rx}$ is fixed at $20 \mathrm{~cm}$ during the B-scan (obtained from $21 \mathrm{~A}$-scans). Antennas are $70 \mathrm{~cm}$ above the pavement in order to collect the backscattered echoes in the far-field. The radar pulse is measured with a metal plane [3]. Since there are two layers, Rx receives two backscattered echoes from the interfaces of pavement. Figure 7 shows the A-scan at the 6 th sample point without air wave and Figure 8 shows the B-scan along the 21 A-scans, within a large frequency band $f \in[0.8,10.8] \mathrm{GHz}$. In order to validate the high resolution power of our proposed method, the used frequency bandwidth for the tested algorithms is $f \in[3.4,4.1] \mathrm{GHz}$ with $M=29$ samples. The product $B \Delta \tau$ is about 0.75 , which indicates the overlapping echoes. According to Figures 7 and 8, the time-delay between the first and second echoes within Layer 1 is about 1.07 ns.

In order to evaluate the performance of the Proposed method-FB and Root-MUSIC-MSSP, several preprocessing techniques are needed: 1) temporal filtering: filter out the air wave; 2) data whitening: divide the raw GPR data by the radar pulse; 3) spatial smoothing: apply MSSP to decorrelate coherent backscattered echoes. Root-MUSIC-MSSP requires all the preprocessing techniques while the Proposed method-FB only 
Future work includes the evaluation of the proposed method on measurements of real roads.

\section{References}

[1] D. J. Daniels, Ground penetrating radar, Wiley Online Library, 2004.

[2] A. Benedetto, L. Pajewski, Civil engineering applications of ground penetrating radar, Springer, 2015.

[3] C. Le Bastard, V. Baltazart, Y. Wang, J. Saillard, Thin-pavement thickness estimation using GPR with high-resolution and superresolution methods, IEEE Transactions on Geoscience and Remote Sensing 45 (8) (2007) 2511-2519. doi: 10.1109/TGRS. 2007.900982.

[4] M. Sun, J. Pan, C. Le Bastard, Y. Wang, J. Li, Advanced signal processing methods for ground-penetrating radar: Applications to civil engineering, IEEE Signal Processing Magazine 36 (4) (2019) 74-84. doi:10.1109/MSP.2019.2900454.

[5] R. Schmidt, Multiple emitter location and signal parameter estimation, IEEE Transactions on Antennas and Propagation 34 (3) (1986) 276-280. doi:10.1109/TAP.1986.1143830

[6] R. Roy, T. Kailath, ESPRIT-estimation of signal parameters via rotational invariance techniques, IEEE Transactions on Acoustics, Speech, and Signal Processing 37 (7) (1989) 984-995. doi:10.1109/29.32276.

[7] C. Le Bastard, V. Baltazart, Y. Wang, Modified ESPRIT (M-ESPRIT) algorithm for time delay estimation in both any noise and any radar pulse context by a GPR radar, Signal Processing 90 (1) (2010) 173-179. doi:10.1016/j.sigpro. 2009.06 .007 
[8] A. L. Swindlehurst, Time delay and spatial signature estimation using known asynchronous signals, IEEE Transactions on Signal Processing 46 (2) (1998) 449-462. doi:10.1109/78.655429.

[9] M. A. Davenport, M. B. Wakin, Analysis of orthogonal matching pursuit using the restricted isometry property, IEEE Transactions on Information Theory 56 (9) (2010) 4395-4401. doi:10.1109/TIT. 2010.2054653.

[10] J. Li, C. Le Bastard, Y. Wang, G. Wei, B. Ma, M. Sun, Enhanced GPR signal for layered media time-delay estimation in low-SNR scenario, IEEE Geoscience and Remote Sensing Letters 13 (3) (2016) 299-303. doi:10.1109/LGRS.2015.2502662.

[11] D. Malioutov, M. Cetin, A. S. Willsky, A sparse signal reconstruction perspective for source localization with sensor arrays, IEEE Transactions on Signal Processing 53 (8) (2005) 3010-3022. doi:10.1109/TSP. 2005.850882.

195 [12] T. Shan, M. Wax, T. Kailath, On spatial smoothing for direction-of-arrival estimation of coherent signals, IEEE Transactions on Acoustics, Speech, and Signal Processing 33 (4) (1985) 806-811. doi:10.1109/TASSP.1985.1164649.

[13] S. U. Pillai, B. H. Kwon, Forward/backward spatial smoothing techniques for coherent signal identification, IEEE Transactions on Acoustics, Speech, and Signal Processing 37 (1) (1989) 8-15. doi:10.1109/29.17496.

[14] F. Han, X. Zhang, An ESPRIT-like algorithm for coherent DOA estimation, IEEE Antennas and Wireless Propagation Letters 4 (2005) 443-446. doi:10.1109/LAWP.2005.860194.

[15] S. Gao, Z. Bao, Data-based matrix decomposition technique for high-resolution array processing of coherent signals, Electronics Letters 23 (12) (1987) 643-645. doi:10.1049/el:19870460

[16] Z. Ye, X. Xu, DOA estimation by exploiting the symmetric configuration of uniform linear array, IEEE Transactions on Antennas and Propagation 55 (12) (2007) 3716-3720. doi:10.1109/TAP. 2007.910493.

$205[17]$ J. A. Cadzow, A high resolution direction-of-arrival algorithm for narrow-band coherent and incoherent sources, IEEE Transactions on Acoustics, Speech, and Signal Processing 36 (7) (1988) 965-979. doi:10.1109/29.1618.

[18] H. Saarnisaari, TLS-ESPRIT in a time delay estimation, in: 1997 IEEE 47th Vehicular Technology Conference. Technology in Motion, Vol. 3, 1997, pp. 1619-1623 vol.3. doi:10.1109/VETEC.1997.605832.

[19] Y. Wang, H. Chen, Y. Peng, Q. Wan, Spatial spectrum estimation theory and algorithm, Tsinghua University, Beijing 25 (2004) $35-55$.

[20] J. A. Cadzow, Y. S. Kim, D. C. Shiue, General direction-of-arrival estimation: a signal subspace approach, IEEE Transactions on Aerospace and Electronic Systems 25 (1) (1989) 31-47. doi:10.1109/7.18659.

[21] J. P. Le Cadre, P. Ravazzola, Approximated stochastic realization and model reduction methods applied to array processing by means of state space models, in: ICASSP '89. IEEE International Conference on Acoustics, Speech, and Signal Processing, 4 (1989) 2601-2604. doi:10.1109/ICASSP.1989.267000.

[22] A. Barabell, Improving the resolution performance of eigenstructure-based direction-finding algorithms, in: ICASSP '83. IEEE International Conference on Acoustics, Speech, and Signal Processing, 8 (1983) 336-339. doi:10.1109/ICASSP. 1983.1172124.

[23] K. Werner, M. Jansson, DOA estimation and detection in colored noise using additional noise-only data, IEEE Transactions on Signal Processing 55 (11) (2007) 5309-5322. doi:10.1109/TSP. 2007.898758.

[24] J. M. Simonin, V. Baltazart, C. Le Bastard, X. Derobert, Progress in monitoring the debonding within pavement structures during accelerated pavement testing on the fatigue carousel, in: 8th RILEM International Conference on Mechanisms of Cracking and Debonding in Pavements, Springer, 2016, pp. 749-755. 


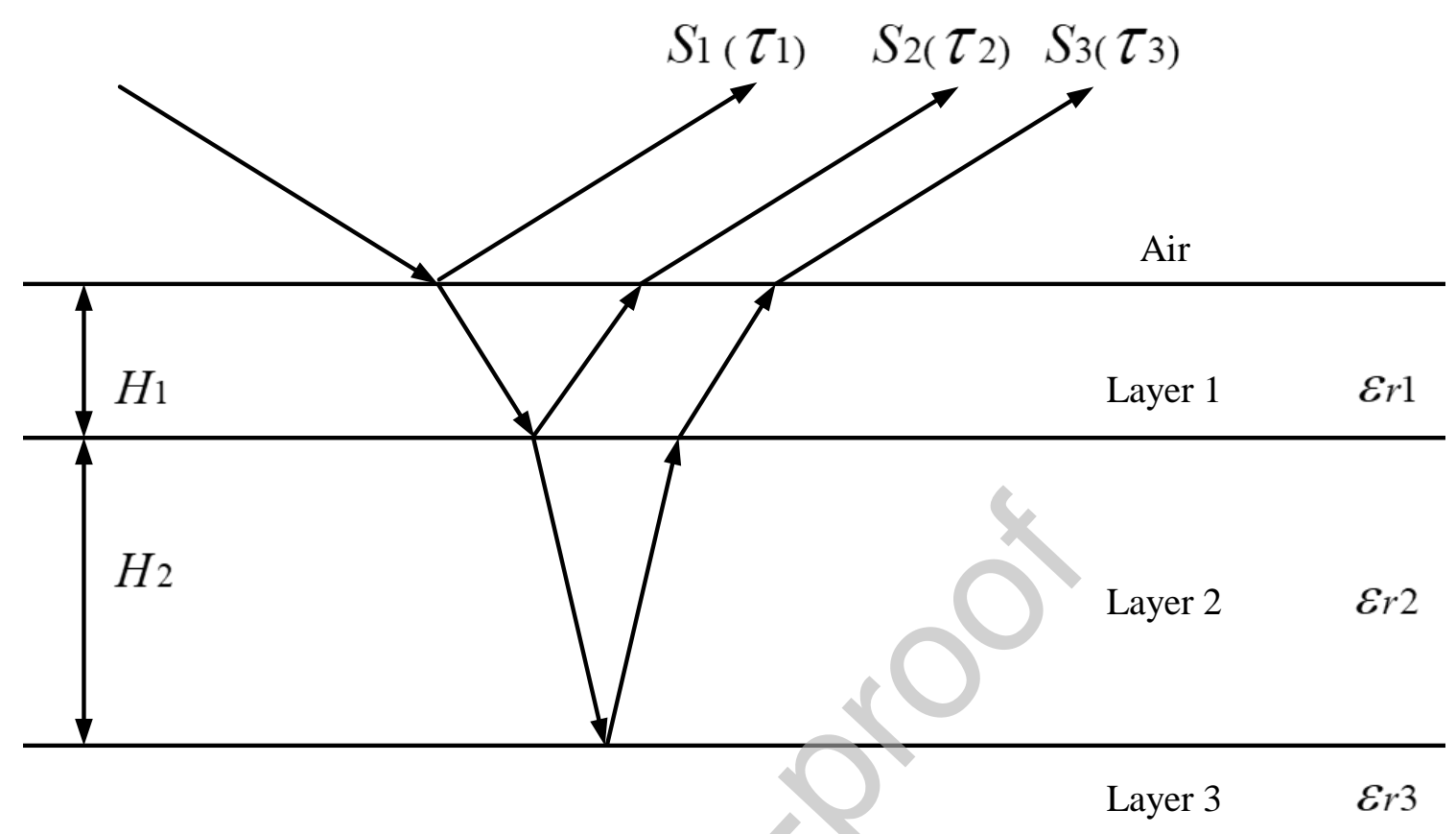

Figure 1: Stratified layers for a simulated setup.

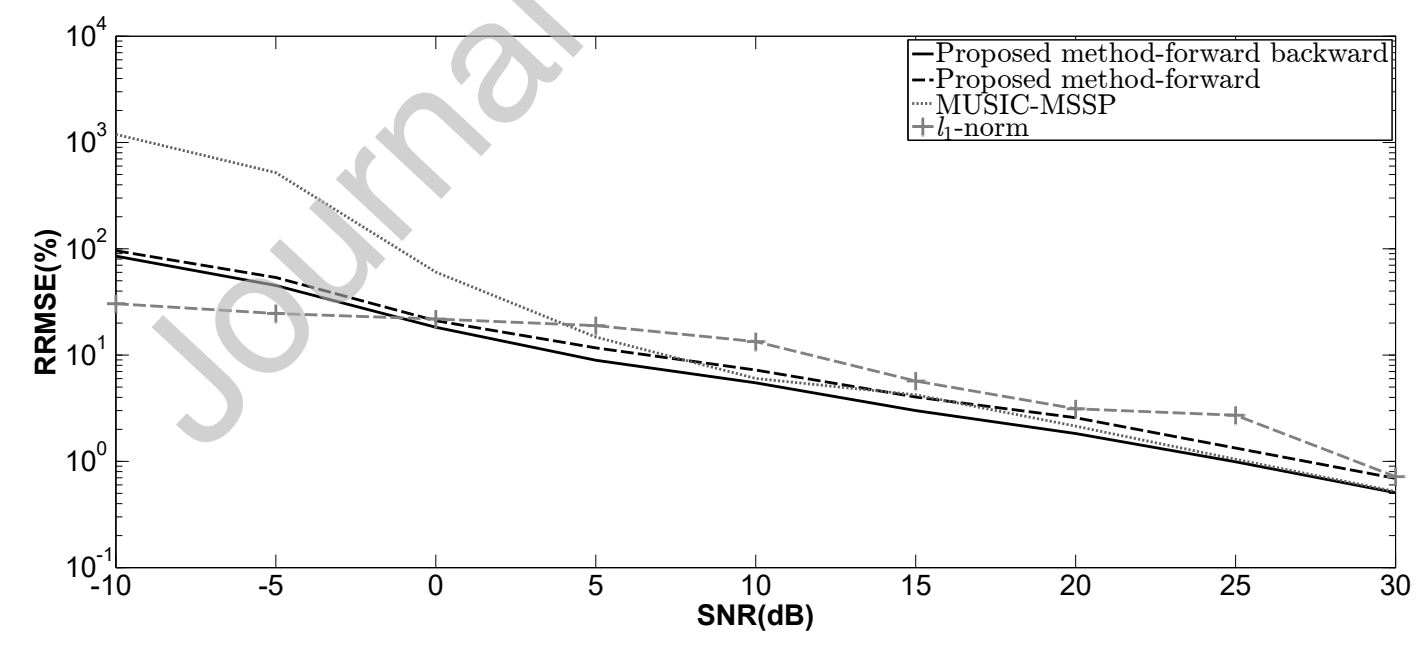

Figure 2: RRMSEs of $\Delta \tau_{1}$ versus SNR. $B \Delta \tau_{1}=0.58$, number of snapshots $=500$. 


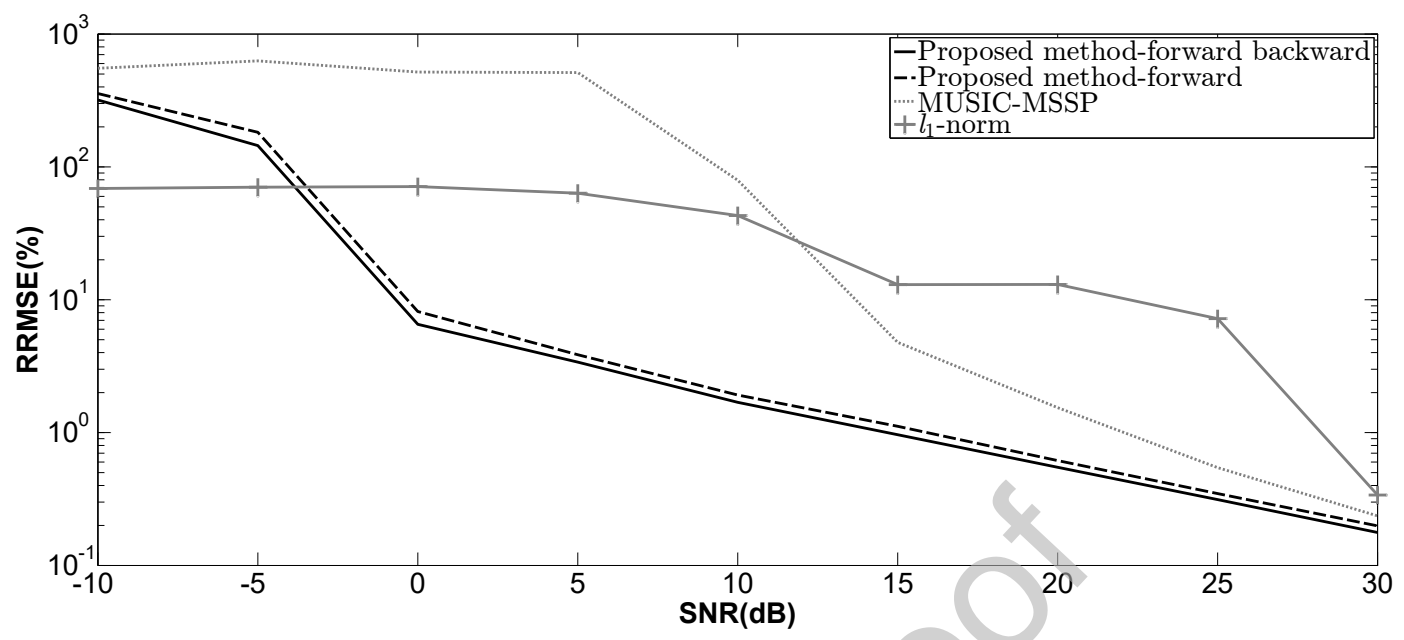

Figure 3: RRMSEs of $\Delta \tau_{2}$ versus SNR. $B \Delta \tau_{2}=1.88$, number of snapshots $=500$.

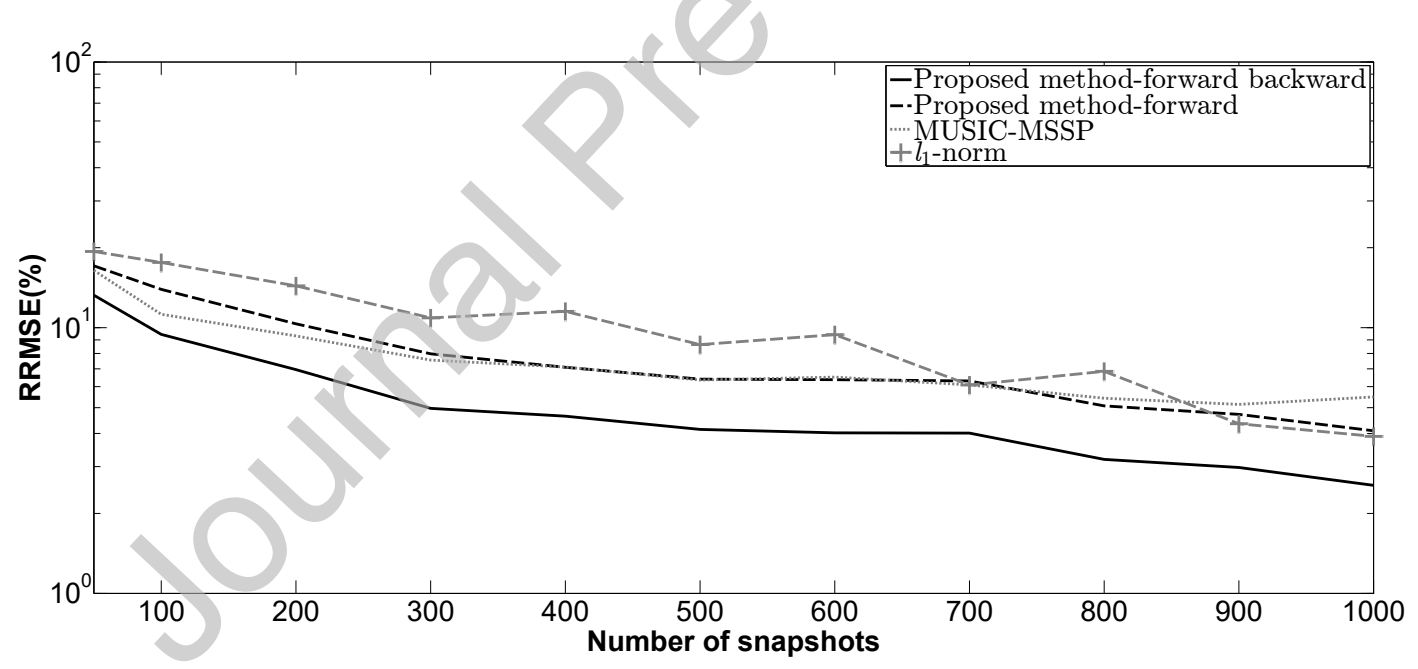

Figure 4: RRMSEs of $\Delta \tau_{1}$ versus the number of snapshots. $B \Delta \tau=0.58, \mathrm{SNR}=15 \mathrm{~dB}$. 


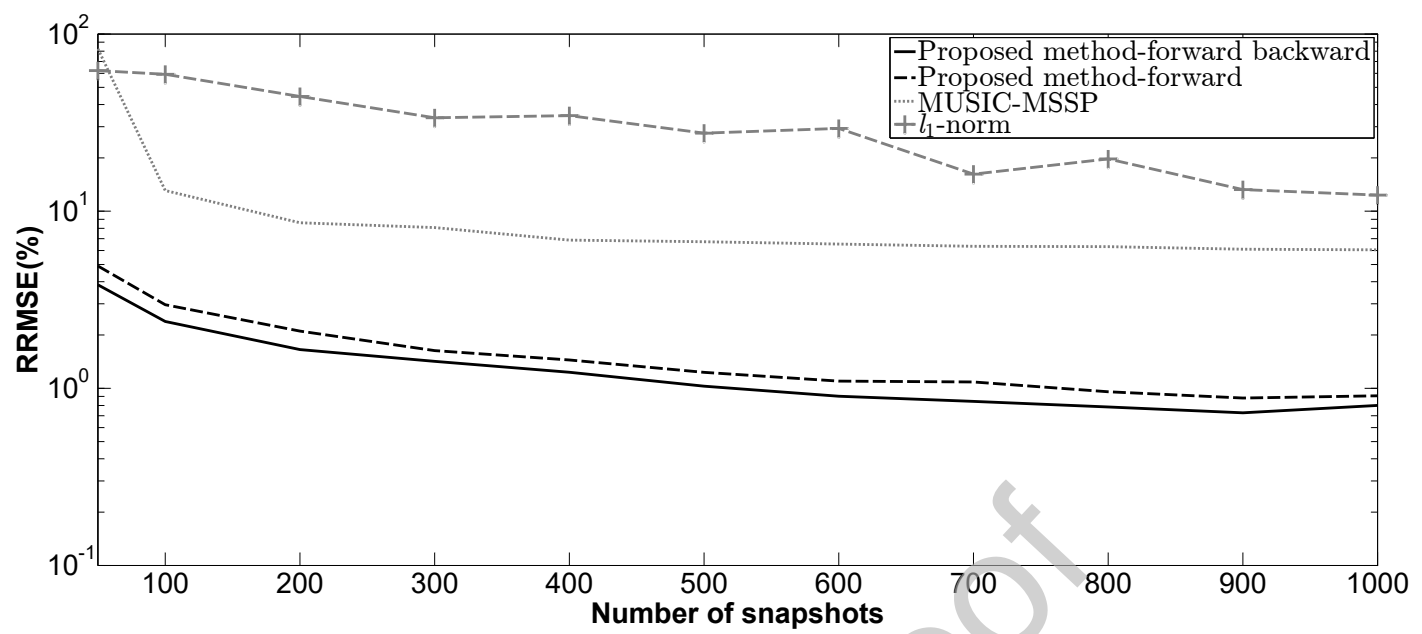

Figure 5: RRMSEs of $\Delta \tau_{2}$ versus the number of snapshots. $B \Delta \tau=1.88, \mathrm{SNR}=15 \mathrm{~dB}$.

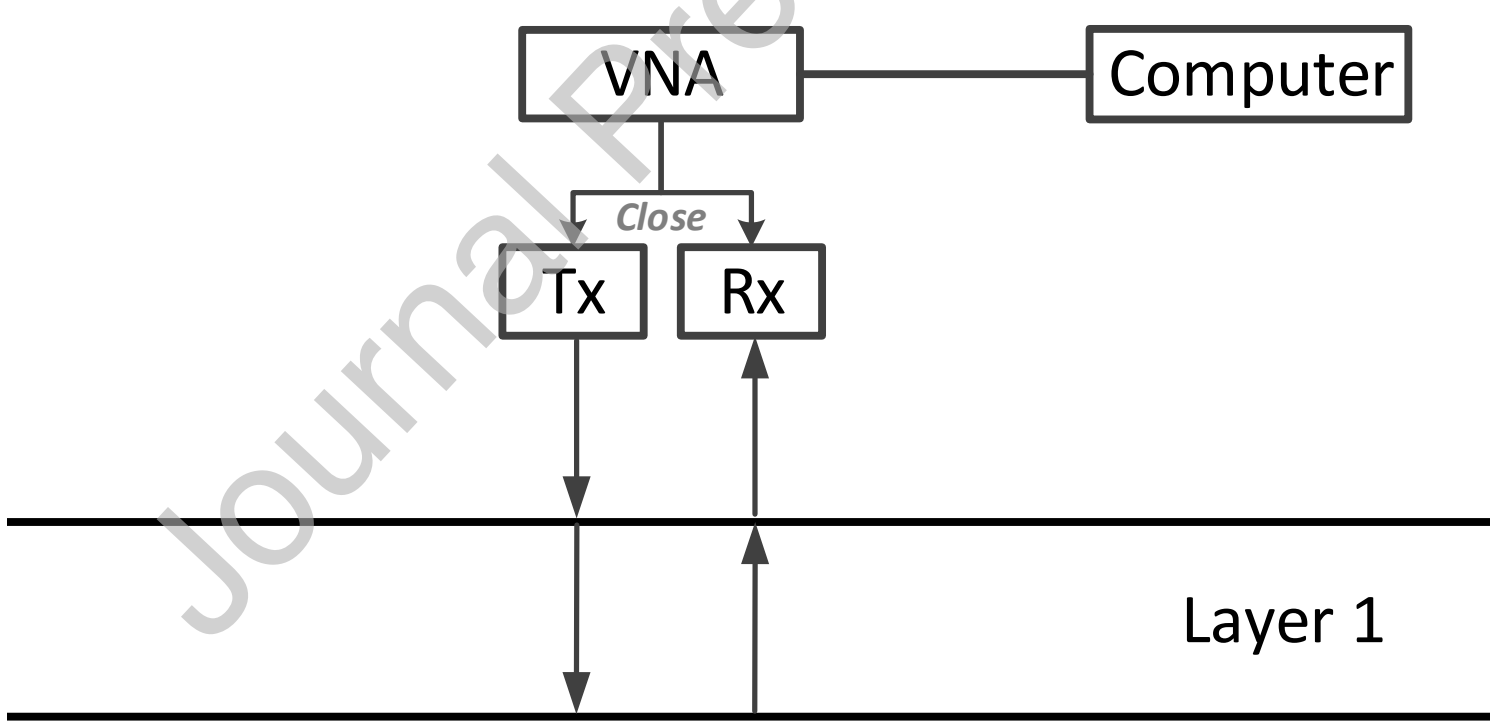

Layer 2

Figure 6: Experimental framework. 


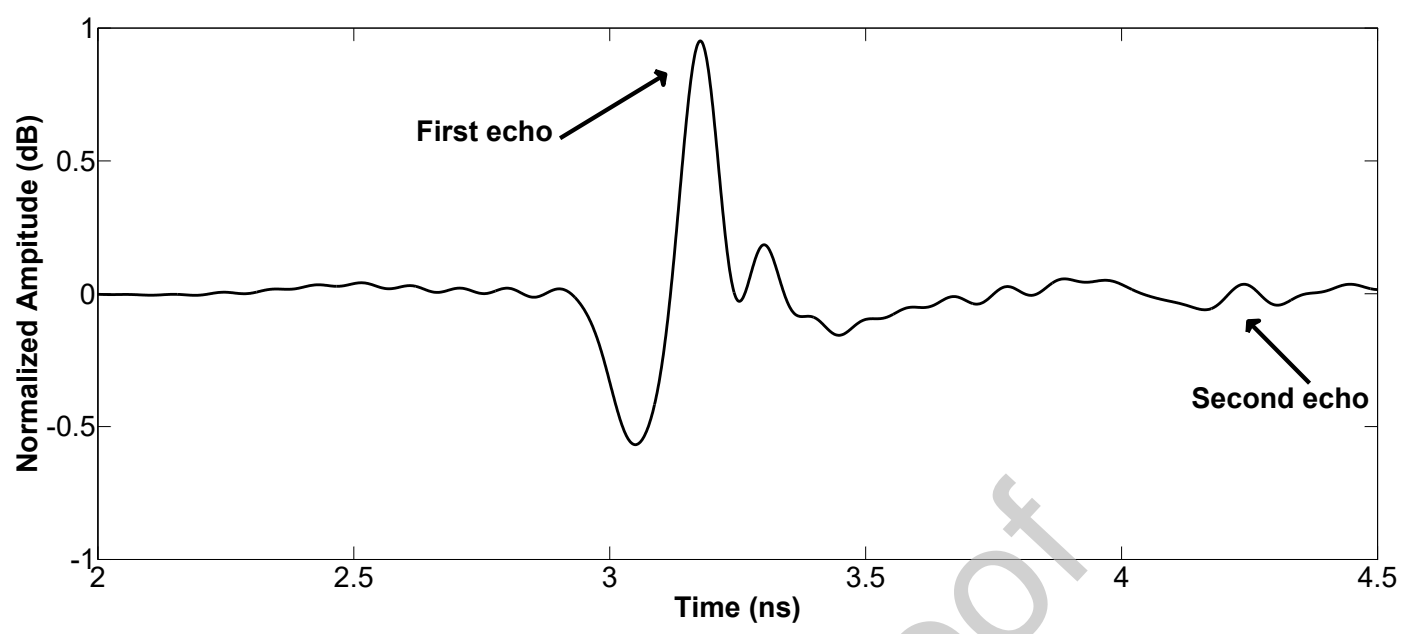

Figure 7: GPR data, A-scan at the 6th sample point within frequency band $(f \in[0.8,10.8] \mathrm{GHz})$.

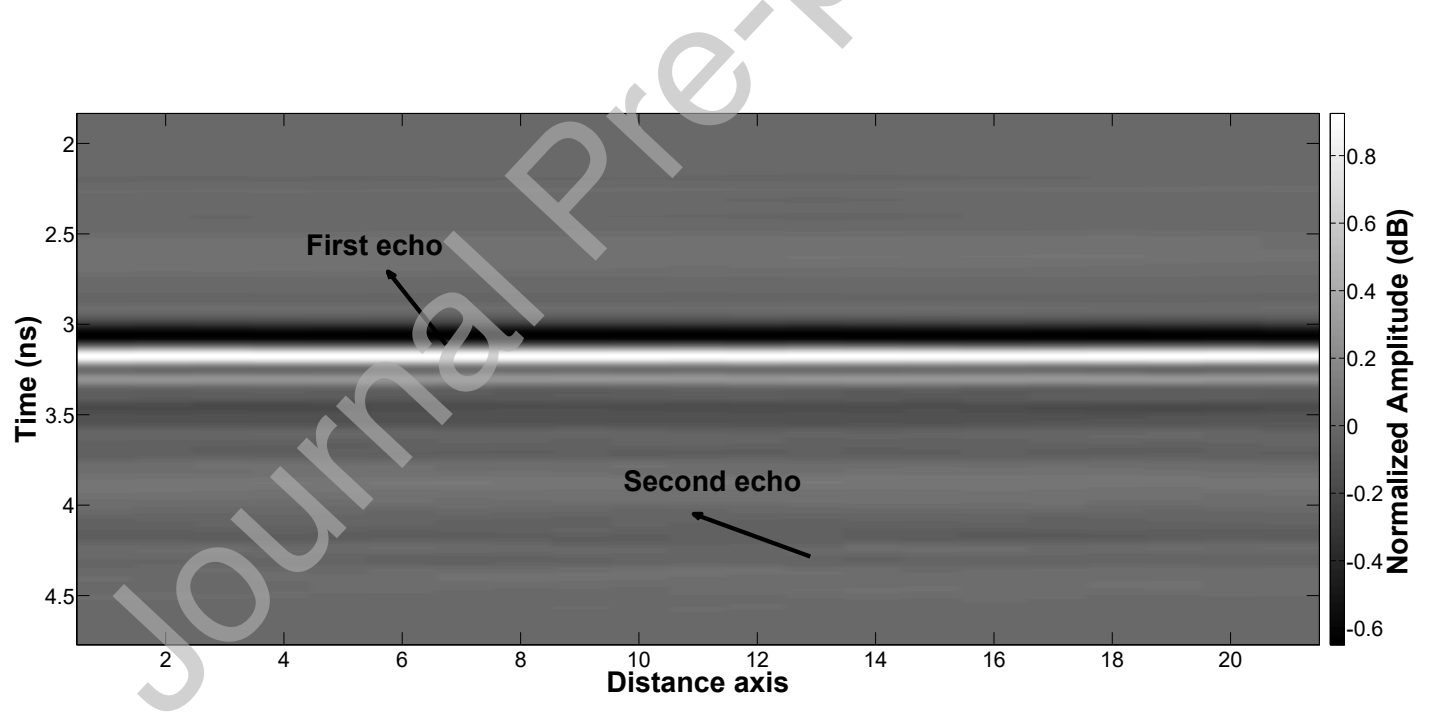

Figure 8: GPR data, B-scan within frequency band $(f \in[0.8,10.8] \mathrm{GHz})$. 
Table 1: Statistical estimation results of the Proposed method-FB and Root-MUSIC-MSSP over 21 A-scans.

\begin{tabular}{lccc}
\hline & Mean $(\mathrm{ns})$ & $\mathrm{Std}^{*}(\mathrm{~ns})$ & Relative error $(\%)$ \\
\hline Proposed method-FB & 1.04 & 0.031 & 3.02 \\
\hline Root-MUSIC-MSSP & 1.13 & 0.055 & 5.80 \\
\hline${ }^{*}$ Standard deviation. & & &
\end{tabular}


Jingjing Pan: Conceptualization, Methodology, Software, Writing - Original Draft Meng Sun: Software, Writing - Original Draft Yide Wang: Methodology, Writing - Review \& Editing Cédric Le Bastard: Resources, Writing - Review \& Editing Vincent Baltazart: Resources, Writing - Review \& Editing 
Conflict of interest statement

We declare that we have no financial and personal relationships with other people or organizations that can inappropriately influence our work, there is no professional or other personal interest of any nature or kind in any product, service and/or company that could be construed as influencing the position presented in, or the review of, the manuscript entitled. 